\title{
II ZWICKY 23 AND FAMILY: A GROUP IN INTERACTION
}

\author{
Elizabeth M. H. Wehner ${ }^{1}$, John S. Gallagher III ${ }^{2}$, Phillip J. Cigan ${ }^{3}$, and Gwen C. Rudie ${ }^{4}$ \\ ${ }^{1}$ Private address; elizabeth@thewehners.net \\ ${ }^{2}$ Department of Astronomy, University of Wisconsin-Madison and 475 North Charter Street, Madison, WI 53706, USA; jsg@astro.wisc.edu \\ ${ }^{3}$ Cardiff University School of Physics and Astronomy Queen's Buildings, The Parade, Cardiff, Cf24 3AA, UK; CiganP@cardiff.ac.uk \\ ${ }^{4}$ The Observatories of the Carnegie Institution for Science and 813 Santa Barbara Street, Pasadena, CA 91101, USA; gwen@obs.carnegiescience.edu \\ Received 2014 September 12; revised 2016 May 28; accepted 2016 June 3; published 2016 September 1
}

\begin{abstract}
II Zw 23 (UGC 3179) is a luminous $\left(M_{B} \sim-21\right)$ nearby compact narrow emission line starburst galaxy with blue optical colors and strong emission lines. We present a photometric and morphological study of II Zw 23 and its interacting companion, KPG103a, using data obtained with the WIYN $3.5 \mathrm{~m}$ telescope in combination with a WFPC2 image from the Hubble Space Telescope archives. II Zw 23 has a highly disturbed outer structure with long trails of debris that may be contributing material toward the production of tidal dwarfs. Its central regions appear disky, a structure that is consistent with the overall rotation pattern observed in the H $\alpha$ velocity field measured from Densepak observations obtained with WIYN. We find additional evidence for interaction in this system, including the discovery of a new tidal loop extending from an associated dwarf galaxy, which appears to be in the process of disrupting along its orbit. We also present $\mathrm{H} \alpha$ equivalent widths and discuss the relative star formation rates across this interacting system.
\end{abstract}

Key words: galaxies: groups: individual (II Zw 23, KPG103a) - galaxies: interactions - galaxies: photometry galaxies: starburst

\section{INTRODUCTION}

II Zwicky 23 (Mkn 1087, UGC 03179) is a blue compact galaxy currently undergoing a global starburst. Although initially thought to have only one companion, KPG103a, López-Sánchez et al. (2004) identified several tidal dwarfs around this system and one dwarf galaxy that appears to predate the tidal dwarf galaxies (TDGs). Several indicators suggest that these group members are currently interacting. The presence of tidally forming dwarfs and tidal streamers of stellar debris all suggest that the enhanced star formation rates (SFRs) in the two main members may have been triggered by an earlier collision with another group member. It is unlikely that KPG103a and II Zwicky 23 have experienced a direct collision with each other. It is more likely that they have been perturbed by a collision with a dwarf companion or had a weak interaction with each other, since there is some evidence of disk structure in both II Zwicky 23 and KPG 103a. While new models by Springel \& Hernquist (2005) suggest that disks may survive if the galaxies are sufficiently gas rich, most simulations suggest that equal mass mergers result in the destruction of the progenitors' disks.

\subsection{Zwicky 23 et al.: Family Traits}

\subsubsection{Zwicky 23}

II Zwicky 23 has cz $\sim 8320 \mathrm{~km} \mathrm{~s}^{-1}$ (Sargent 1970) and lies at a distance of $115 \mathrm{Mpc}$ (for $H_{0}=72 \mathrm{~km} \mathrm{~s}^{-1} \mathrm{Mpc}^{-1}$ ). It is embedded in an $\mathrm{HI}$ cloud of $M_{\mathrm{HI}}=1.9 \times 10^{10} M_{\odot}$, with a corresponding dynamical mass of $1.8 \times 10^{11} M_{\odot}$ (Gordon \& Gottesman 1981), though more recent work by López-Sánchez et al. (2004) find a Keplerian mass of $\sim 5 \times 10^{10} M_{\odot}$. The SFR of this luminous blue compact galaxy is clearly enhanced (Hunter \& Gallagher 1985), though the exact SFR is unknown. The $1.4 \mathrm{GHz}$ radio data (Condon et al. 2002; López-Sánchez et al. 2004) suggest an SFR $\sim 4.65 M_{\odot} \mathrm{yr}^{-1}$ for $M>5 M_{\odot}$ but including stars down to $M>0.1 M_{\odot}$ brings the $\mathrm{SFR}_{1.4 \mathrm{GHz}}$ up to $25.8 M_{\odot} \mathrm{yr}^{-1}$. The far-infrared fluxes (Kennicutt 1998; López-
Sánchez et al. 2004) yield an SFR $\sim 11.5 M_{\odot} \mathrm{yr}^{-1}$, and LópezSánchez et al. (2004) estimate an $\mathrm{SFR}_{\mathrm{H} \alpha}=4.5 M_{\odot} \mathrm{yr}^{-1}$ from their long-slit spectroscopy.

López-Sánchez et al. (2004) find an inclination for II Zwicky 23 of $i=37^{\circ}$ and that the northern side is moving away from us, while the southern side is approaching us. In this case, the tidal debris tails emanating from the right of II Zwicky 23 in Figure 1 are polar extensions, and the debris to the north is roughly in the plane of the disk.

\subsubsection{KPG103a}

KPG103a is the less well-studied companion of II Zwicky 23. It lies $81 \mathrm{kpc}$ to the southwest and has a very similar velocity $\left(\mathrm{cz}=8313 \mathrm{~km} \mathrm{~s}^{-1}\right)$ (Marzke et al. 1996). It has been classified as an SB, H II galaxy and Marzke et al. (1996) suggest the presence of a weak two-armed spiral structure. In optical images, there appear to be three knots in the center of the galaxy, possibly the nucleus and a circumnuclear ring, or multiple nuclei left over from an as of yet unrelaxed collision. There also appear to be some diffuse, low surface brightness extensions. These properties, along with what appears to be an enhanced SFR, suggest that KPG103a has recently undergone some type of tidal interaction.

\subsubsection{Tidal Debris, Dwarfs, and Knots}

López-Sánchez et al. (2004) perform a thorough analysis of the knots and regions of interest in II Zwicky 23. We have created our own labeling scheme, but have provided their numerical labels as well in Figure 1 for comparison. The regions have been overlaid on our $B$-band image. López-Sánchez et al. (2004) suggest, based on their finding of metallicities above the expected $\mathrm{L}-\mathrm{Z}$ relationship, that Regions $\mathrm{J}$ and $\mathrm{M}$ are TDGs. They also propose that Region $\mathrm{C}$ may be a giant $\mathrm{H}$ II region, rather than an infalling galaxy, as originally proposed by Keel (1988). Region J has a particularly high SFR, but both Regions $\mathrm{J}$ and $\mathrm{M}$ contain significant populations of older stars (López-Sánchez et al. 2004). While not noted as being tidal dwarfs, Regions 11 and L, along the polar extensions, do show signs of some new star formation in 


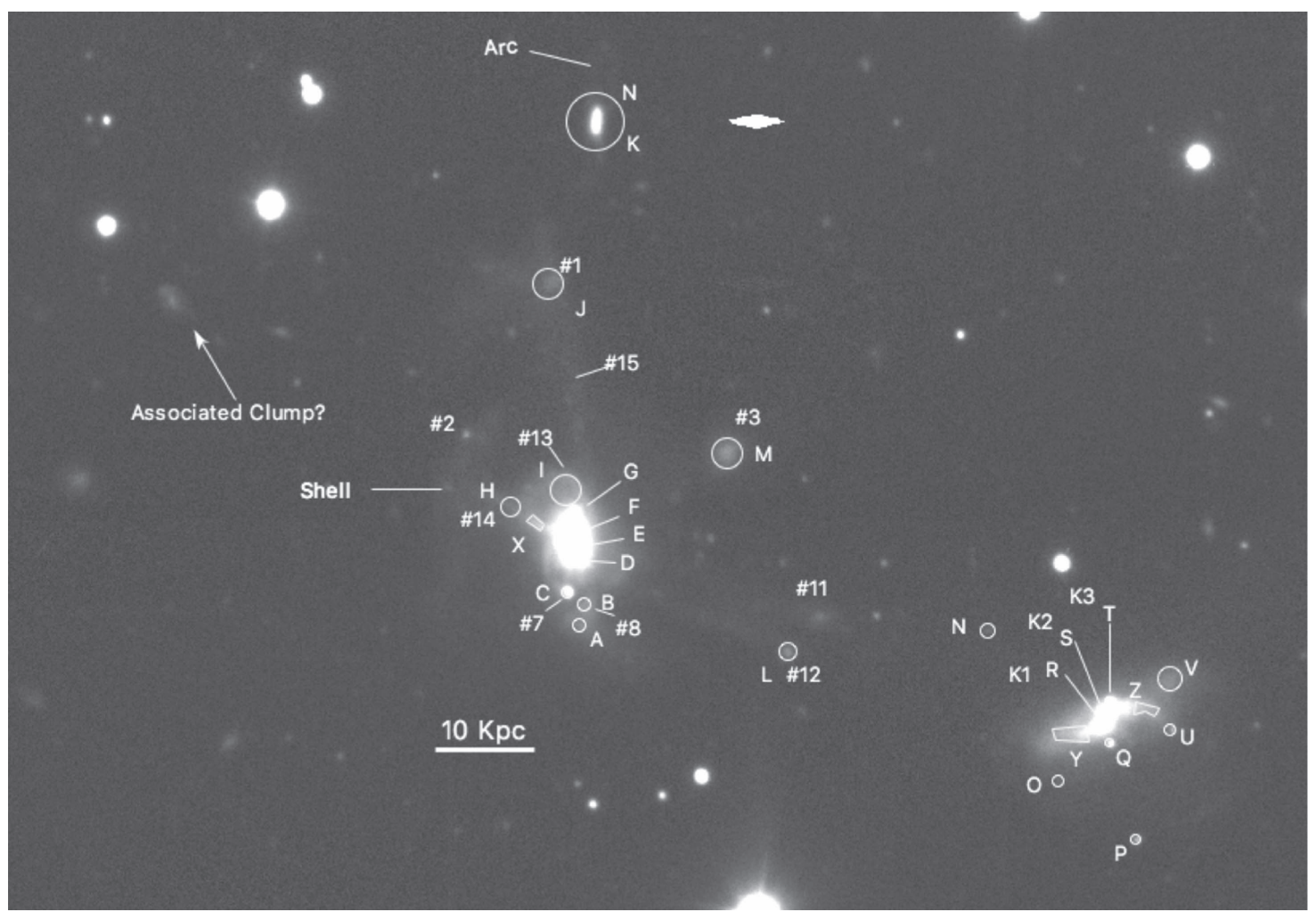

Figure 1. Deep $B$-band image of II Zwicky 23 and its companions. The image was taken on the WIYN 3.5 m telescope in Kitt Peak, Arizona.

addition to what appears to be material stripped from II Zwicky 23 (López-Sánchez et al. 2004).

They also find that Region $\mathrm{K}$ has a distinct rotational pattern and a lower $[\mathrm{O} / \mathrm{H}]$ ratio than expected for a tidally forming galaxy. They therefore propose that $\mathrm{K}$ is an independent group member, not formed as a result of any recent collision.

\section{OBSERVATIONS AND REDUCTIONS}

\subsection{Broadband Imaging}

We observed II Zwicky 23 and its companions using the MiniMosaic camera on the WIYN 3.5 m telescope in Kitt Peak, Arizona. Data were taken on two different runs. On 2000 November 17, we obtained broadband $B$ and $R$ data, as well as narrowband $\mathrm{H} \alpha$ images taken with the W17 filter. These data were taken under photometric conditions and standard stars (Landolt 1992) were observed at the beginning and the end of the night. On 2002 November 2, we obtained $V$-band and deeper $B$ band data. Conditions were not photometric for this run, and patchy clouds were present in the sky throughout the night.

All data were bias-subtracted and flat-fielded in IRAF. ${ }^{5} \mathrm{We}$ then used MIDAS ${ }^{6}$ to remove the cosmic rays from each frame and to fit the sky background and subtract it. These frames were then combined in IRAF using pixel maps to eliminate the gap between the two CCDs that comprise the MiniMosaic camera and to eliminate dead pixels.

To calibrate the data, we used the $B$ - and $R$-band data from 2000 November 17 to obtain a photometric solution and then

\footnotetext{
5 IRAF is distributed by the National Optical Astronomy Observatories, which are operated by the Association of Universities for Research in Astronomy, Inc., under cooperative agreement with the National Science Foundation.

6 Munich Image Data Analysis System, provided by the European Southern Observatory (ESO).
}

measured the zeropoint for the $B$ - and $R$-band images. We then used these zero points to find magnitudes of several field stars in the 2000 November 17 data, which we then used to calibrate the $B$-band image from 2002 November 2 . The $V$-band images lacked photometric standard stars and could not be calibrated. In all we obtained $1700 \mathrm{~s}$ in $B, 800 \mathrm{~s}$ in $\mathrm{V}, 500 \mathrm{~s}$ in $R$, and $2000 \mathrm{~s}$ in $\mathrm{H} \alpha$.

\subsection{Narrowband Imaging}

The $\mathrm{H} \alpha$ images were bias-subtracted, flat-fielded, cosmic-ray corrected, sky subtracted, and combined in the same way as the broadband data. Once a combined $\mathrm{H} \alpha$ image was obtained, we scaled the $R$-band image using stars in the field of view and the PSFMATCH function in IRAF and subtracted it from the $\mathrm{H} \alpha$. This yielded the final, continuum-subtracted $\mathrm{H} \alpha$ image. Attempts were made to flux-calibrate the $\mathrm{H} \alpha$ image, but standard stars and calibration targets were insufficient in number and a final calibration could not be achieved. In order to examine the relative SFRs, we then created an equivalent width image by dividing the continuum-subtracted $\mathrm{H} \alpha$ image by the $R$ band. The resulting image can be seen below in Figure 5 and is discussed in Section 3.2.

\subsection{DensePak IFU Spectroscopy}

We observed II Zwicky 23 on 2005 February 9 using the DensePak instrument at the Cassegrain focus of the WIYN $3.5 \mathrm{~m}$ telescope. Conditions were not photometric, but transparency was good. We obtained $2200 \mathrm{~s}$ total, centered around the $\mathrm{H} \alpha$ line. Data were reduced and combined using the CCDRED and the DOHYDRA packages in IRAF. We also obtained two arcs of the CuAr lamp for wavelength calibration. 

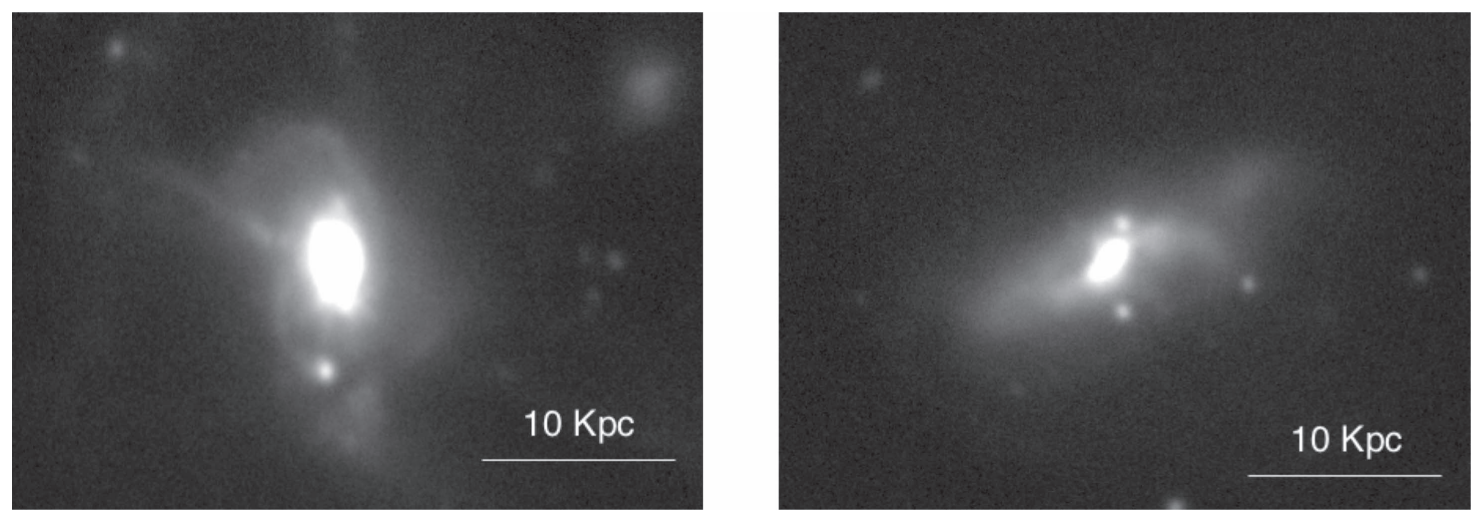

Figure 2. $B$-band image of II Zwicky 23 (left) and KPG103a (right).

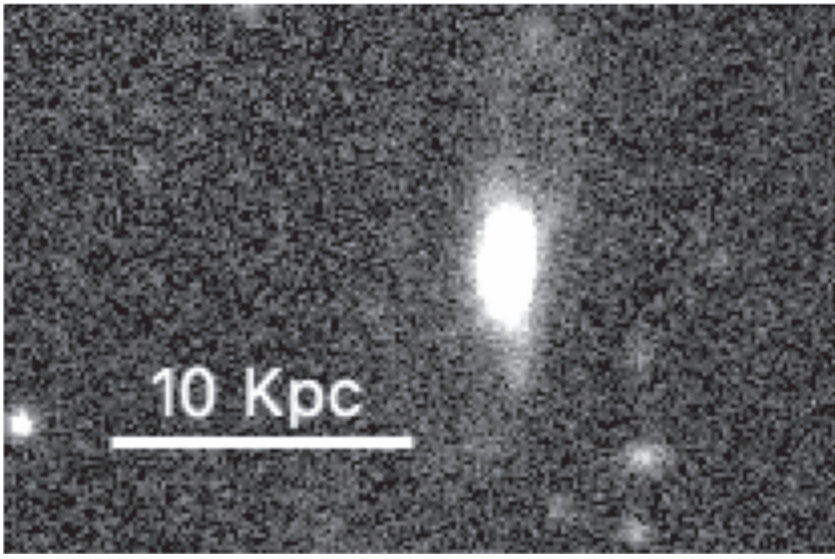

Figure 3. Region "K" directly to the north of II Zwicky 23. Previous work (López-Sánchez et al. 2004) suggests that this is a dwarf galaxy that existed prior to the latest burst of star formation. This deep $B$-band image reveals a stellar loop of material extending to the north. As such, this dwarf galaxy appears to be in the process of disrupting along its orbit around II Zwicky 23.

\section{RESULTS}

\subsection{Broadband Imaging}

In addition to those described in previous works, there are several new and interesting features in our deep $B$-band image, shown in Figure 1. The first is the small, stellar arc, marked in Figure 1. If the orientation is as suggested in López-Sánchez et al. (2004), then this arc of stars likely sits in the plane of the disk, and may be a disk ripple or shell. For an inclination of $37^{\circ}$, the inner edge of this ripple lies approximately $18 \mathrm{kpc}$ from the center of II Zwicky 23. There is also a stellar extension that appears to connect the center of II Zwicky 23 with the shell (also labeled in Figure 1). These stars could be the remains of a disrupted companion galaxy, which plunged through II Zwicky 23 and created the shell. Considering the uncertainties in projection, it could extend out of the plane of the disk, unconnected to the disk ripple. It is interesting to note the presence of stellar clumps further out, if one draws a line continuing this stellar extension. These clumps lie at distances (uncorrected for projection) of 35 and $42 \mathrm{kpc}$ from II Zwicky 23. These regions are not visible in the $\mathrm{H} \alpha$ image, so if they are at the same redshift, then they have no detectable ionized gas. Without further data, we cannot conclusively determine whether these clumps are associated with this group.
In Figure 1, there are also a number of tidal streamers surrounding the main body of II Zwicky 23. One extends to region $\mathrm{K}$ and two to Regions 11 and $\mathrm{L}$. The weak spiral structure is also visible in Figure 2(a), arcing around the south to Region $\mathrm{C}$ and on the northern side as well. One can also see the beginnings of what might be debris leading out to Region $\mathrm{M}$.

KPG103a is shown in Figure 2(b). This galaxy also exhibits many signatures of interaction. In the $B$-band image there does appear to be a weak two-armed spiral pattern, though it is somewhat distorted. The northeastern arm curves briefly clockwise, before proceeding to turn counter-clockwise. While the other arm is more well-behaved, there is an obvious diffuse stellar clump to its immediate upper right. This clump could be the remains of a merged galaxy or part of KPG103a's own disk that was pulled out by tidal forces during an interaction. This disk has clearly been disrupted by a collision with another galaxy.

Also interesting are the two bright and roughly circular knots in the center of this galaxy. These clumps may also contain the remains of a merged galaxy, and may even be its nucleus. If we are seeing double nucleii due to an as yet unrelaxed merger, then the interaction most likely occurred within the last $\mathrm{X}$ Gigayears. KPG103a also has a larger, more diffuse low surface brightness component extending from the west/northwest. This can be seen in Figure 5. We propose that this material may have been pulled from KPG103a during a tidal interaction, but follow-up observations are required to confirm.

Another feature of note is in the neighborhood of the dwarf galaxy, K. In our deep $B$-band image, there is a visible loop extending from Region $\mathrm{K}$ to the north. This loop is shown in more detail in Figure 3. This loop is visible in all our continuum wavebands and is therefore most likely a loop of stellar material. It is not detected in our $\mathrm{H} \alpha$ image, and thus appears to contain little or no ionized gas. One possible interpretation is that this dwarf galaxy companion is in the process of disrupting, leaving stars in its wake. In this case, the northern tip of the loop may be where $\mathrm{K}$ reached its peak distance from II Zwicky 23 and turned around in its orbit. If we then use this peak as its maximum orbital distance from II Zwicky 23 and assume a simple orbit, then its last close encounter with II Zwicky 23 was 1.4 Gyr ago-a time much too distant to have caused the current central starburst, which is thought to be only 6-9 Myr old (López-Sánchez et al. 2004).

In addition, we find weak evidence of a stellar bridge (Figure 1) between KPG103a and II Zwicky 23. This bridge is only visible in our $B$-band image, which is our deepest image and deeper data in other wavebands would be required to confirm its existence. 


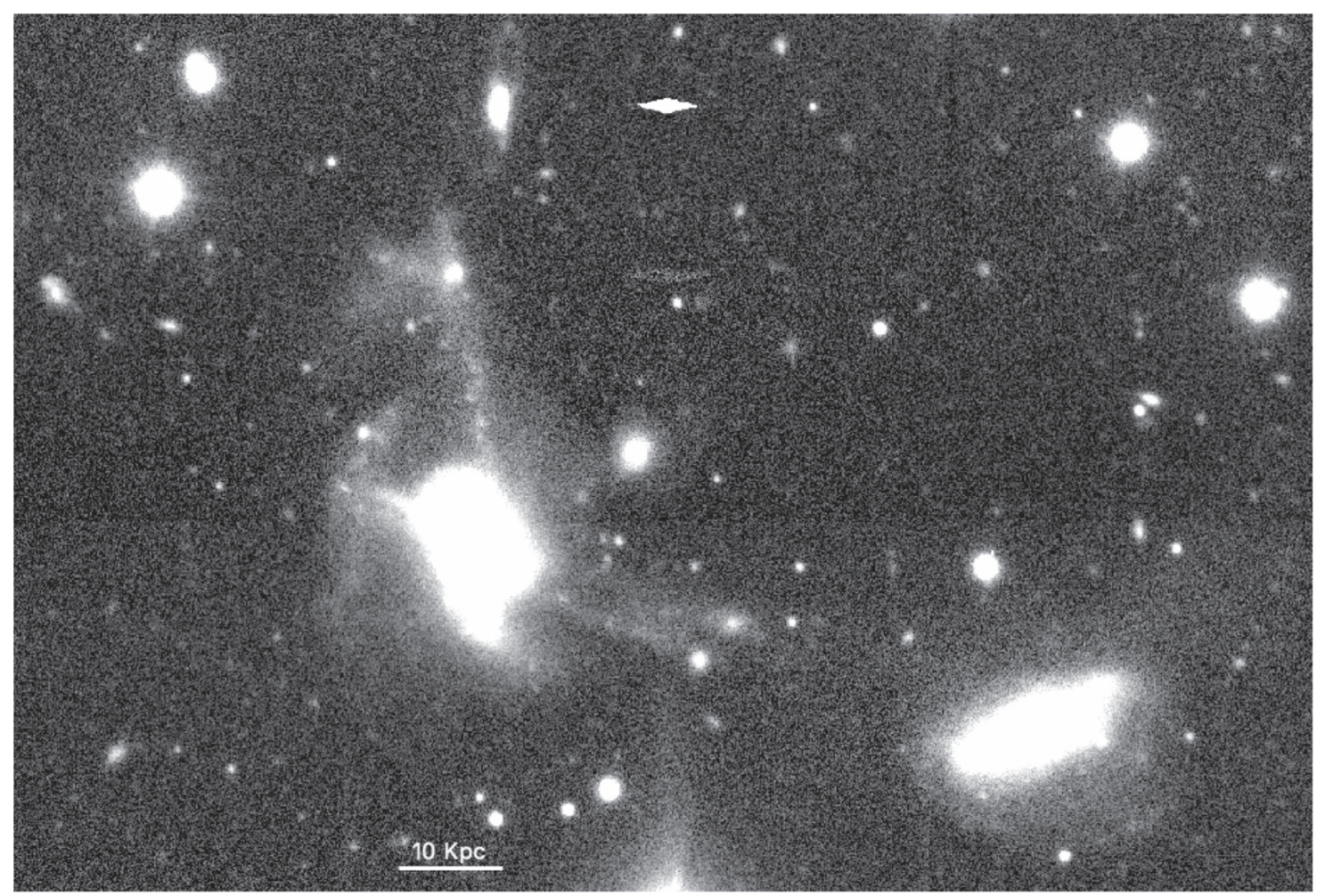

Figure 4. Deep $B$-band image shown in logarithmic scale to emphasize the faintest tidal debris features in this system.
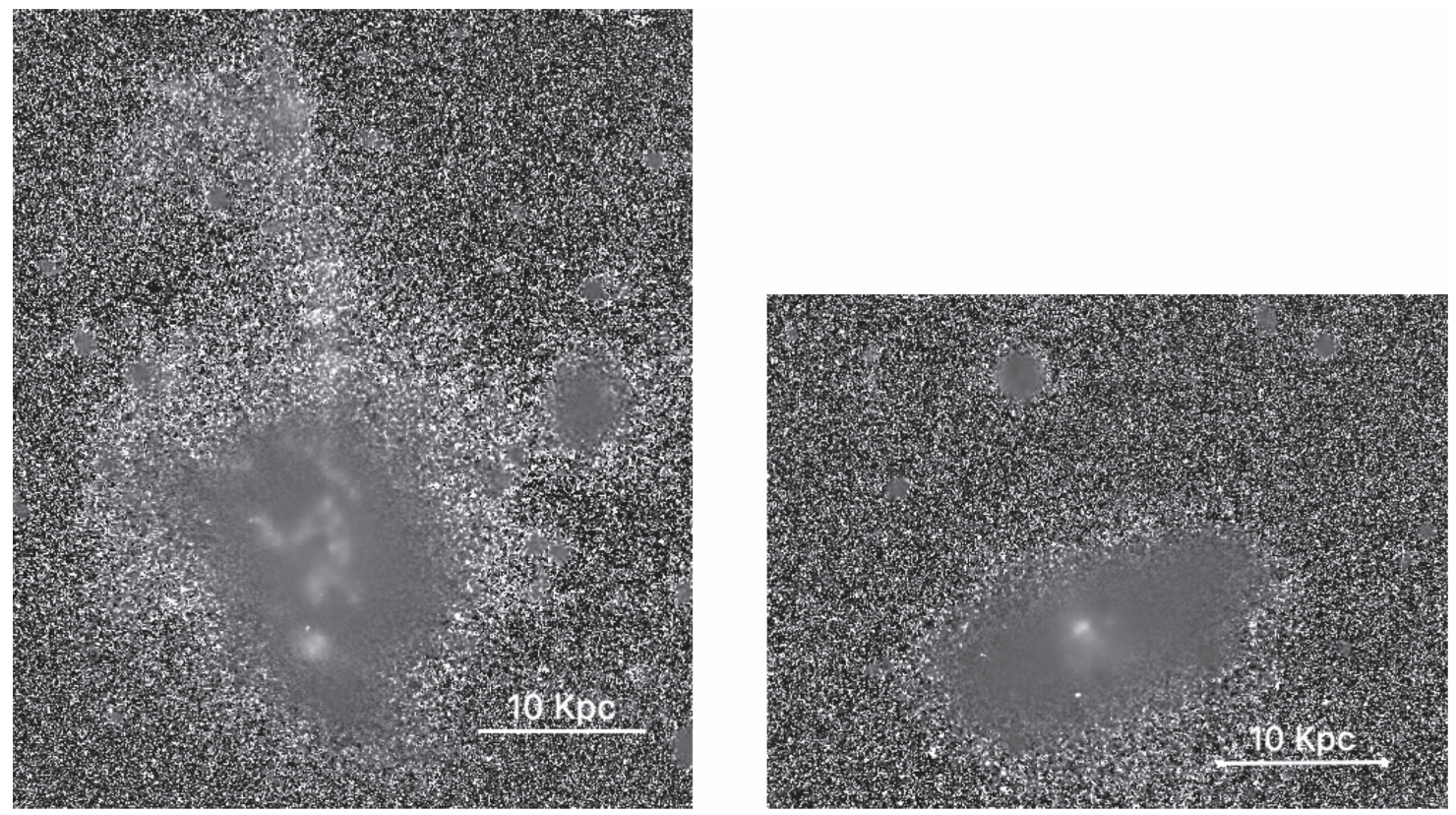

Figure 5. H $\alpha$ equivalent width of II Zwicky 23 (left; with extensions up to Region K and KPG103a, right). Images are on the same physical scale.

\subsection{Narrowband Imaging}

Long-slit spectroscopy has been done to measure $\mathrm{H} \alpha$ at various position angles, but $\mathrm{H} \alpha$ narrowband imaging is useful to examine the star formation in $2 \mathrm{D}$ across the system. We used the MiniMosaic camera on the WIYN $3.5 \mathrm{~m}$ telescope to obtain deep narrowband imaging of this system.

Figure 5 shows an image of the equivalent width (EW) structure of both II Zwicky 23 and KPG103a. These images represent the $\mathrm{H} \alpha$ emission from each galaxy and have been continuum subtracted and then normalized to the continuum by dividing by the $R$-band image. In the resulting images, the more brighter the region, the more intense the ongoing star formation.

As can be seen in Figure 5, $\mathrm{H} \alpha$ emission is located across the disk in II Zwicky 23. Clumpy star formation also appears to extend all the way out to Region $\mathrm{K}$ in the north (the disrupting dwarf galaxy), which appears to have particularly intense $\mathrm{H} \alpha$ emission. KPG103a also exhibits star formation, though it is 
Table 1

Magnitudes and Equivalent Widths for Regions in II Zwicky 23

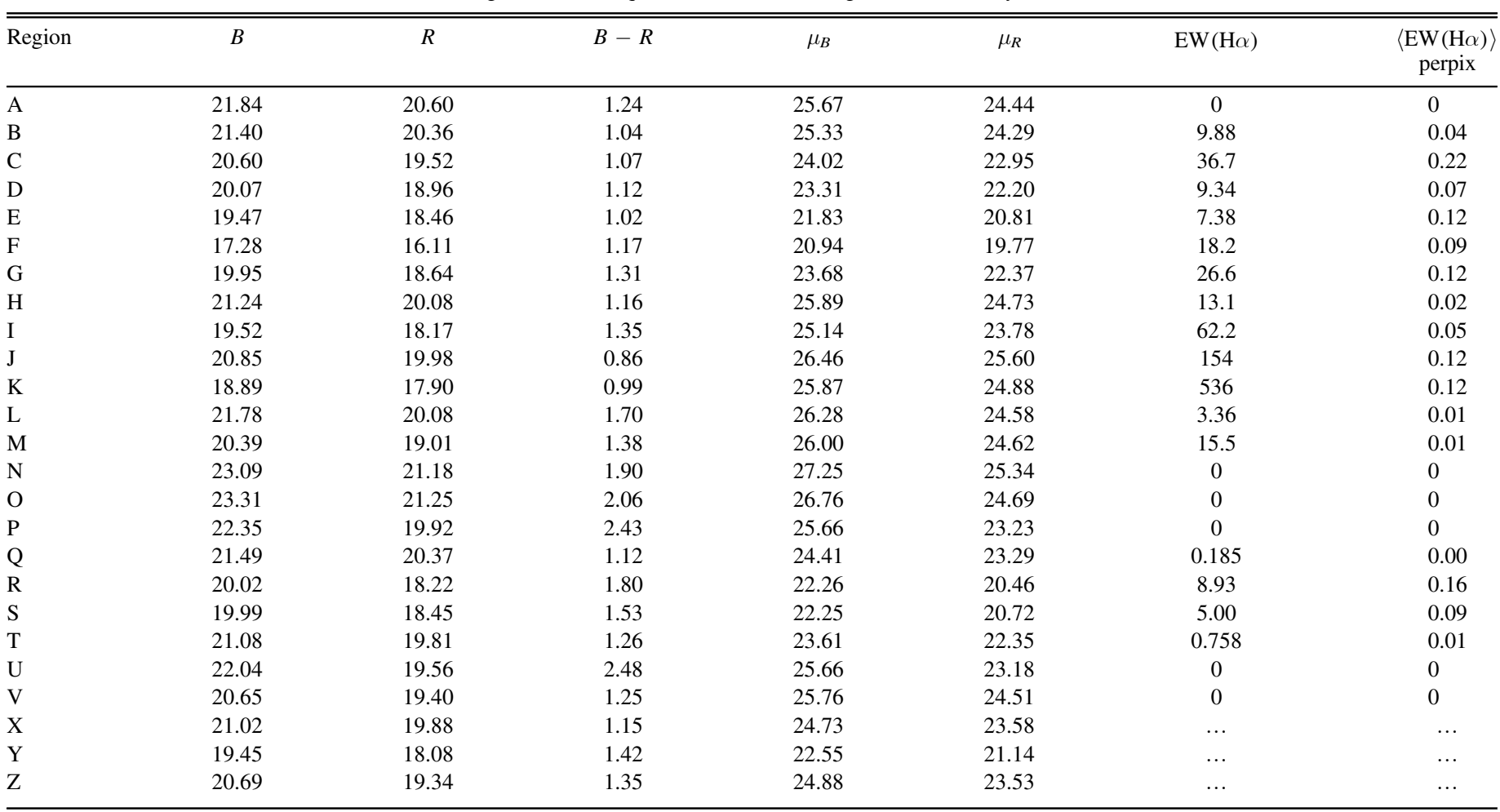

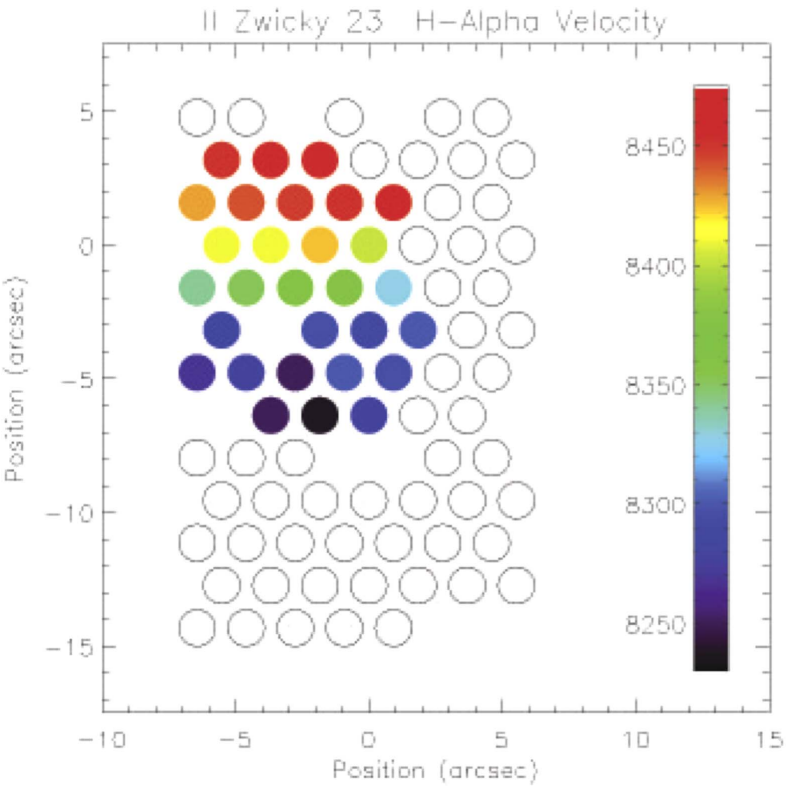

Figure 6. DensePak data for II Zwicky 23 centered on the $\mathrm{H} \alpha$ line. The velocity for each fiber is represented by its color.

more limited in extent with only the inner $4^{\prime \prime}$, or $2 \mathrm{Kpc}$, detected in $\mathrm{H} \alpha$.

The EWs of each region defined in Figure 1 can be found in Table 1. These were measured by summing up the total flux in each aperture. Since the regions vary significantly in size, Table 1 also lists the EW per pixel for each region. This latter value indicates the relative strength of the star formation in each location, without being affected by the size of the aperture.

The $\mathrm{H} \alpha$ structure, as seen in Figure 5, is lumpy and does not entirely coincide with the location of the spiral arms in II Zwicky 23. The star forming regions seem to trail off in an odd curve with the faint stellar debris toward the northeast. We also note that the possible TDG of region $\mathrm{M}$ does appear to be experiencing some star formation, with a modest EW of $15.5 \mu \mathrm{ms}$, while the other TDG candidate (region J) shows signs of a more intense starburst. Region $\mathrm{K}$, the disrupting dwarf galaxy to the north, also appears to be undergoing intense star formation, with a similar EW/pixel as Region J.

The most intense star formation is occuring in Region C. This region is noted as a large H II region in Keel (1988). López-Sánchez et al. (2004) support this conclusion, although their plot of $[\mathrm{O} / \mathrm{H}]$ versus $M_{B}$ indicates that this region may also be a TDG. Their conclusion is based on the lack of central concentration and no detected kinematic deviation from II Zwicky 23.

\subsection{DensePak}

The DensePak array contains 91 fibers, each of which spans 3 arcsec. The entire array covers $30 \times 40 \operatorname{arcsec}$ on the sky. For each fiber with detectable $\mathrm{H} \alpha$, we fit a Gaussian to the line to find the location of its peak. From this, we calculated the velocity of the gas in each $3^{\prime \prime}$ fiber. We then assigned the velocity range to a color table and plotted the velocity for each fiber, as shown in Figure 6. Despite its obvious signs of disturbance in the stellar component (as evidenced by the clumpy, asymmetrical disk and extended tidal debris filaments), we find a fairly smoothly rotating disk, supporting II Zwicky 23's classification as a spiral disk galaxy. We measure a $\Delta v=233 \mathrm{~km} \mathrm{~s}^{-1}$ across the disk, or a maximum rotation 
speed of $v_{\text {rot }} \sim 117 \mathrm{~km} \mathrm{~s}^{-1}$. It is possible that this number is an overestimate, since it includes fibers such as that on Region $\mathrm{C}$, which is the point in the disk moving most rapidly toward us. As such, this Region $\mathrm{C}$ may be an $\mathrm{H}$ II region at the tangent point at its radius.

Another possible explanation of Region $\mathrm{C}$ is that we are seeing the result of a small galaxy interacting with the disk of II Zwicky 23. It is possible that debris from a companion galaxy is moving through the disk, inducing intense star formation in the disk along the way.

\section{DISCUSSION}

II Zwicky 23 and its companions are a group in turmoil; there is now extensive evidence that this system is being affected by numerous collisions. The disruption of Region K, the ongoing starburst, and tidal arms of debris extending out of the disk all suggest ongoing interactions between the members of this group. In addition, the presence of shells in II Zwicky 23, as well as the possible double nuclei in KPG103a, support the idea that collisions with former dwarf companions have recently occurred. However, despite all this, the ionized component of II Zwicky 23's disk has maintained a smoothly rotating structure. This system is an excellent example of intragroup interactions driving galaxy evolution and merits future study. In particular, additional wavebands would allow for comparison of the stellar populations within II Zwicky 23 and its companions with spectral synthesis models. Additionally, a well-calibrated $\mathrm{H} \alpha$ image would serve to solve the debate as to the intensity of star formation in this system.

E.H.W., J.S.G., P.J.C., and G.C.R. thank the National Science Foundation for support through grant NSF AST9803018 to the University of Wisconsin and the University of Wisconsin-Madison Graduate School for additional funding provided by the Wisconsin Alumni Research Foundation. We would also like to thank Bill Harris for his comments on an early draft of this paper. This research has made use of the NASA/IPAC Extragalactic Database (NED), which is operated by the Jet Propulsion Laboratory, California Institute of Technology, under contract with the National Aeronautics and Space Administration. We would also like to thank the referee for helpful and insightful comments.

\section{REFERENCES}

Condon, J. J., Cotton, W. D., \& Broderick, J. J. 2002, AJ, 124, 675

Gordon, D., \& Gottesman, S. T. 1981, AJ, 86, 161

Hunter, D. A., \& Gallagher, J. S. 1985, AJ, 90, 1457

Keel, W. C. 1988, A\&A, 202, 41

Kennicutt, R. C. 1998, ApJ, 498, 541

Landolt, A. 1992, AJ, 104, 340

López-Sánchez, Á R., Esteban, C., \& Rodríguez, M. 2004, A\&A, 428, 425

Marzke, R. O., Huchra, J. P., \& Geller, M. J. 1996, AJ, 112, 1803

Sargent, W. L. W. 1970, ApJ, 160, 405

Springel, V., \& Hernquist, L. 2005, ApJL, 622, L9 